\title{
A Hybrid Model for Explaining Older Adult' Continuance Intention toward SNSs
}

\author{
Wei Liu \\ Jiangxi Key Laboratory of Data and Knowledge Engineering \\ Institute of Information Resources Management \\ School of Information Technology \\ Jiangxi University of Finance \& Economics, \\ Nanchang 360013, P. R. China \\ Lw0519@126.com
}

\begin{abstract}
Due to the rapid growth of social net services (SNSs), research into SNSs continuance usage has recently emerged as an important issue in information systems adaption. This study develops an integrated model based on the expectation-confirmation model (ECM), the technology acceptance model (TAM), and the theory of planned behavior (TPB), and apply them into the context of older adults' continuance intention toward SNSs. The hypothesized model is validated empirically using a sample collected from 250 older adults who had prior experience with SNSs and was tested against the proposed research model using structure equation modeling. Analysis results demonstrate that satisfaction has the most significant effect on older adults' continuance intention, followed by perceived usefulness, attitude, subject norm and perceived behavioral control. The results of these findings for SNSs practitioners are discussed at the end of this work.
\end{abstract}

Keywords: Older adults; continuance intention; Adoption model

\section{Introduction}

Nowadays, older adults make up the fastest growing consumer segment of Internet users [1]. They are enhancing their independence by accessing online services such as banking, shopping, or healthcare management, and Social network services (SNSs) including recreation and communication.

SNSs can be defined as an individual web page which enables online humanrelationship building by collecting useful information and sharing it with specific or unspecific people[1, 2]. Although older adults have begun using SNSs more frequently and many research have implied the expansion of the SNSs could enhance the quality of life of older adults, internet-using older adults have not adopted this communication medium nearly as quickly as younger adults [2, 3, 4]. The wide ranging consequences for these differences between young and old may include challenges for intergenerational communication, for information sharing and learning [2]. Without mediated communication channels that both groups feel comfortable using, valuable connections may be lost [2]. So, more and more study seeks to understand what factors encourage or discourage older adults from using SNSs $[1,2,4,5]$.

On the other hand, many older adults discontinue using SNSs after initially accepting it, their intention to continue using such system is still very low. Although initial acceptance of SNSs is an important first step toward achieving SNSs success, actual success still needs continued usage rather than on first-time use. Therefore, understanding the factors affecting older adults' intention to continue using SNSs 
will assist developers and vendors in designing popular contents and strategies that are more likely to increase the older adults' use of SNSs.

In this paper, we synthesize the expectation-confirmation model (ECM), the technology acceptance model (TAM) and theory of planned behavior (TPB) to explain older adults' intention to continue using SNSs. We combine these three models for the following two reasons: First, while ECM is a rigorous model that predicts the key factors of IS continuance [6], it employs only three variables, namely satisfaction, confirmation, and post-adoption expectations to explain behavioral intention [2]. However, a older adult's behavioral intention toward adopting SNSs will also be affected by other factors, such as the opinions of important individuals (subjective norms), and even if older adults have a strong intention to perform, they may feel that they lack the necessary resources and skills (perceived behavioral control). The TPB theory can address this gap. Second, though TPB captures the roles of individuals, organizational members, and social influences on behavioral intention, it does not inform us what attitudinal beliefs would affect a user's attitude [7]. According to Taylor's research, TAM provides two attitudinal beliefs, namely perceived ease of use and perceived usefulness as two major antecedents of attitude, which make up precedent factors of attitude for TPB [7].

Prior research has examined ECM, TAM, and TPB independently in explaining information technology usage. So, some researches suggest that these models independently provide a partial understanding of users' cognitive processes related to IT usage. It is therefore possible that, when combined, these theories may collectively provide an improved and more comprehensive understanding of the cognitive processes and behaviors related to IT usage than when each theory considered alone[2, 6, 7]. The primary contributions of this study are its examination of the integration of ECM, TAM and TPB in explaining the older adult's continuance usage intention of SNSs and an empirical evaluation of which factors are critical to affecting this intention. The findings from this paper may therefore help bridge the existing research gap between acceptance and continuance usage of the older adults. The results of this work are expected to give practitioners an increased understanding of older adult's continuance intention, which can then be used as a guideline to devise more appropriate SNSs products for older adults.

The rest of the paper is organized as follows. Section 2 presents our research model and hypotheses, while Section 3 proposes the measurement method and scales. We present the research results in Section 4, followed by discussion in Section 5. Finally, the limits of this work are presented in Sections 6.

\section{Research Model and Hypotheses}

\subsection{Expectation-Confirmation Model (ECM)}

The ECM is widely used to explore user behavior in an IS post-adoption environment [6]. The ECM posits that an individual's intention to continue IS usage is dependent on three variables: the user's level of satisfaction with the IS, the extent of user's confirmation of expectations, and perceived usefulness. User satisfaction refers to an overall evaluation of an IS, which reflects an emotion-based response about the target IS [7]. Empirical evidence on IS continuance supports that user satisfaction is a major factor of IS continuance intention [6, 7]. So, we give the first hypotheses associated with older adult's satisfaction with SNSs usage.

H1: Older adult's satisfaction with SNSs usage positively affects their continued SNSs usage intention. 
According the ECM, user's satisfaction with IS is determined by the user' confirmation of expectations and their perceived usefulness of IS. Older adults develop initial expectations about SNSs before using it. After the older adults actually use SNSs, they update their expectations of SNSs performance based on their direct experiences. When SNSs outperforms relative to their initial expectations, their post-adoption expectations are confirmed. Otherwise, their postadoption expectations are disconfirmed. The level of confirmation and post adoption expectations affect the level of older adults' satisfaction about the IS. Moreover, the IS adoption literature has consistently found that perceived usefulness is the most important determinant of users' adoption intentions [6, 7]. Perceived usefulness is the user's beliefs about how useful a piece of technology is for achieving a certain goal. Where technology can be used to enhance job performance, perceived usefulness may play a significant role in encouraging adoption of new systems. As a result, the ECM posits users' perceived usefulness of IS has a positive effect on their intention to continue IS usage. Because SNSs is a kind of information technology on the Internet, we derived the following hypotheses from the ECM:

H2. Older adult's confirmation of expectations positively affects their satisfaction with SNSs.

H3. Older adult's perceived usefulness of SNSs positively affects their satisfaction with SNSs.

H4. Older adult's perceived usefulness of SNSs positively affects their continued SNSs usage intention.

H5. Older adult's confirmation of expectations positively affects their perceived usefulness of SNSs.

\subsection{The Technology Acceptance Model (TAM)}

Davis proposed TAM to explain the potential user's behavioral intentions of using a technological innovation. The TAM is based on the Theory of Reasoned Action and Theory of Planned Behavior which proposes two particular beliefs including perceived usefulness and perceived ease of use. Perceived usefulness is defined as "the degree to which a person believes that using a particular system would enhance his/her job performance", and perceived ease of use is defined as "the degree to which a person believes that using a particular system would be free of physical and mental effort" [2]. Further, perceived usefulness and perceived ease of use both affect a person's attitude toward using the system, and consistent with TRA, these attitudes toward using the system determine behavioral intentions, which in turn lead to actual system use [2]. So, the older adult users need to treat SNSs as a useful tool that can improve their life quality, enable them to better communicate with their friends, family and others online. Both perceived usefulness and perceived ease of use are beliefs that, according to TRA, will affect older adults' attitude. So, we propose the next hypothesis:

H6. Perceived usefulness positively affects older adult's behavioral attitude toward SNSs.

H7. Perceived ease of use positively affects older adult's behavioral attitude toward SNSs.

In addition, TAM states that perceived ease of use affects behavioral intention indirectly through perceived usefulness (Davis, 1989). That is, perceived usefulness mediates the effect of perceived ease of use on behavioral intention. Many empirical studies have supported this argument [2]. Thus, we posit that:

H8. Perceived ease of use positively affects older adult's perceived usefulness of SNSs. 


\subsection{Theory of Planned Behavior (TPB)}

The theory of planned behavior (TPB) was developed based on the theory of reasoned action (TRA), which argues that both behavioral attitude and subjective norm affect behavioral intention, which in turn affects the actual behavior. TPB adds the perceived behavioral control to TRA, which affects behavioral intention and actual behavior [8]. Many studies have investigated these three constructs and found that they are valid in explaining individual intention to use various forms of IT [7].

In our model, we propose that both perceived behavioral control and subjective norm will impact the continuance intention. Subjective norm refers to "the perceived social pressure to perform or not to perform the behavior" [8]. In other words, subjective norm is related to the normative beliefs about the expectation from other people. Many older adults choose to use SNSs because their friends or relatives are the users of SNSs system, and they recommend it to them. Hence, we propose:

H9. Subjective norm positively affects older adult's continued SNSs usage intention.

Perceived behavioral control refers to "people's perception of ease or difficulty in performing the behavior of interest". It is associated with beliefs about the presence of control factors that may facilitate or hinder the performance of the behavior in question (Liao, Chen, et al., 2007). In this case, although SNSs can enhance the older adult' independence and some other benefits, they still need to have the basic internet skills to use it. Thus, we posit that:

H10. Perceived behavioral control positively affects older adult's continued SNSs usage intention.

Attitude refers to "the degree of a person's favorable or unfavorable evaluation or appraisal of the behavior in question" [2]. According to the TPB, attitude impacts users' behavioral intention, which in turn influences their actual behavior. When individuals form positive attitude towards SNSs, they will have a stronger intention toward adopting it, and thus they are more likely to use it. Recently, some studies have viewed users' continuance and acceptance decisions as the same as acceptance decisions [12]. Therefore, the following hypothesis is proposed.

H11. Behavioral attitude toward SNSs positively affects older adult's continued SNSs usage intention.

\section{Research Methodology}

\subsection{Instrument Development}

We used a questionnaire survey to test our theoretical model. Each item corresponding to the constructs was measured using a seven-point Likert scale, with answer choices ranging from "disagree strongly" (1) to "agree strongly" (7). The survey items for the constructs were adapted from pre-validated ECM, TAM and TPB studies. The question measurements were reworded to fit the older adults and SNSs environment. The scale items for perceived usefulness, perceived ease of use and attitude were adapted from Davis [9], subjective norm and perceived behavioral control were adapted from Taylor and Todd [10]. The continuance intention, satisfaction and confirmation were adapted from Bhattacherjee [6]. The survey items are listed in Appendix A.

Before conducting the survey, the survey measurements were reviewed by five IS experts to identify problems in the wording, content, and to remedy any ambiguities 
in the questions. After several modifications of the items based on the suggestions from these IS experts, the modified questionnaire was pilot-tested on 56 older adults. The results of the pilot test were evaluated by using Cranach's reliability and factor analysis. The reliability coefficient was first calculated for the items of each construct, and the standard lower bound for Cranach's alpha set at 0.7 (Anderson \& Gerbing, 1988). A factor analysis was then performed to examine whether the items produced the anticipated number of factors and whether the individual items were loaded on their appropriate factors. All items had high loadings on their related factors and low cross-loadings on other factors, showing good convergent and discriminate validities.

\subsection{Subjects and Data Collection}

Empirical data was collected via a paper-based survey from two communities in Shang Hai located in eastern China. A total of 250 older adults aged 50-65 years participated in this study. All participants reported using the SNSs or internet services at least once a week.

Each participant was given a survey booklet or a link to complete the survey online. Participants were told, "This study is designed to understand more about how older adults feel about social networking websites." If participants were not currently users of SNSs, but had internet experience, they were instructed to "answer based on their thoughts about SNSs, rather than their experience using them." Each survey began with a table presenting basic information about SNSs, as well as specific information about some popular social networking platforms. To encourage participation, participants were given some gifts, funds provided by the author.

\section{Results}

In analyzing the collected data, we followed the two-step approach of SEM, which is closest in spirit to Byoungsoo Kim [7]. First, a confirmatory factor analysis (CFA) was used to measure convergent and discriminant validity. Then, we examined the structural model to investigate the strength and direction of the relationships among the theoretical constructs.

\subsection{Measurement Model}

A CFA was conducted to test the measurement model. This study assessed model fit in terms of four different indices: root mean square error approximation (RMSEA), comparative fit index(CFI), non-normed fit index (NNFI), and standardized root mean square residual (SRMR). The four fit indices are recommended based on their relative stability and insensitivity to sample size [7]. As shown in Table 1, all recommended fit indices were within the recommended level, representing a good model fit. This study calculated convergent validity, reliability, and discriminate validity of the measurement scales [7]. First, to assess convergent reliability, this study checked item loadings. Convergent validity can be satisfied if item loadings are 0.60 or higher [7]. The lowest loading of this study was 0.812 , confirming the convergent validity. Second, the CR and AVE values were calculated to check reliability. The reliability was acceptable if the CR value was 0.70 or higher and the AVE value was 0.50 or higher. As shown in Table 2, all factors met both criteria for acceptable reliability. Lastly, the assessment of discriminate validity was conducted by comparing the shared variances between factors with the AVE of the individual factors. Table 3 gives the inter-construct correlations and the square roots of the AVEs. This table shows that the square root 
of the AVE was higher than their shared variances. The results confirmed the discriminate validity of the model constructs.

\section{Table 1. Summary of Fit Indices}

\begin{tabular}{llcl}
\hline Fit indices & Recommended value & Measure model & Structural model \\
\hline$x^{2} / \mathrm{df}$ & $\leqslant 5$ & 3.25 & 2.59 \\
RMSA & $\leqslant 0.08$ & 0.067 & 0.076 \\
CFI & $\geqslant 0.90$ & 0.98 & 0.95 \\
NNFI & $\geqslant 0.90$ & 0.95 & 0.71 \\
SRMR & $\leqslant 0.10$ & 0.049 & 0.079 \\
\hline
\end{tabular}

\subsection{Structural Model and Hypothesis Testing}

The eleven hypotheses presented above were tested collectively using the structural equation modeling (SEM) approach, also tested using LISREL 8.5. As shown in Table1, the same set of fit indices was used to test the fit of the structural model. All the indices suggested a fairly good fit. The results of the SEM are presented in Fig. 1.

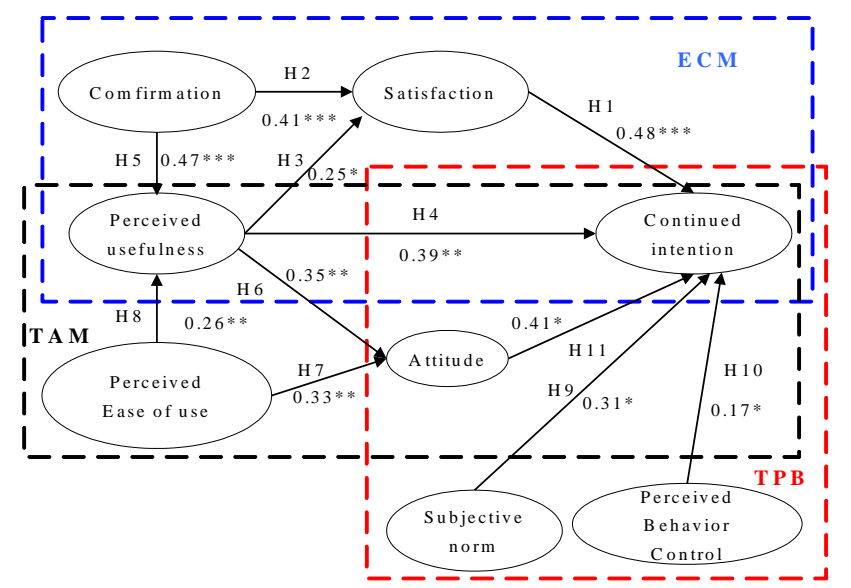

\section{Figure 1. Research Model and Analysis Results}

In this study, user satisfaction was found to play a significant role in older adults' SNSs continuance intention. Consistent with our expectations, continuance intention was found to be significantly related to perceived usefulness. Therefore, $\mathrm{H} 1$ and $\mathrm{H} 4$ are accepted. Also, perceived usefulness was found to have a significant effect on older adults' satisfaction, $\mathrm{H} 3$ is accepted too. The effects of confirmation of expectations on perceived usefulness and user satisfaction are all confirmed. Therefore, H2 and H5 are supported. Perceived ease of use positively affects the perceived usefulness, and they are both positively associated with the attitude. Hence, H6, $\mathrm{H} 7$ and $\mathrm{H} 8$ are also accepted. The effect of attitude, perceived behavioral control and Social norm on continuance intention are confirmed, H9, H10 and H11 are all accepted. 
Table 2. Scale Reliabilities

\begin{tabular}{|c|c|c|c|c|c|c|}
\hline Construct & Item & Mean & Standard deviation & Loading & $\mathrm{CR}$ & AVE \\
\hline \multirow[t]{3}{*}{ Continuance intention } & CI1 & 3.89 & 1.58 & 0.906 & 0.92 & 0.8364 \\
\hline & $\mathrm{CI} 2$ & 3.44 & 1.59 & 0.905 & & \\
\hline & $\mathrm{CI} 3$ & 3.76 & 1.66 & 0.927 & & \\
\hline \multirow[t]{4}{*}{ User satisfaction } & US1 & 3.72 & 3.65 & 0.925 & 1.02 & 0.9116 \\
\hline & US2 & 3.78 & 3.8 & 0.992 & & \\
\hline & US3 & 3.65 & 3.53 & 0.954 & & \\
\hline & US4 & 3.83 & 3.72 & 0.913 & & \\
\hline \multirow[t]{3}{*}{ Perceived usefulness } & PU1 & 4.18 & 1.8 & 0.911 & 0.99 & 0.7887 \\
\hline & PU2 & 4.4 & 1.99 & 0.941 & & \\
\hline & PU3 & 3.77 & 1.61 & 0.895 & & \\
\hline \multirow[t]{3}{*}{ Confirmation } & CM1 & 3.6 & 1.37 & 0.844 & 0.94 & 0.6741 \\
\hline & CM2 & 3.33 & 1.25 & 0.845 & & \\
\hline & CM3 & 3.23 & 1.33 & 0.812 & & \\
\hline \multirow[t]{3}{*}{ Attitude } & AT1 & 3.74 & 1.25 & 0.894 & 0.94 & 0.7612 \\
\hline & AT2 & 3.88 & 1.23 & 0.898 & & \\
\hline & AT3 & 3.94 & 1.56 & 0.899 & & \\
\hline \multirow[t]{3}{*}{ Perceived ease } & PE1 & 4.38 & 1.66 & 0.891 & & \\
\hline & PE2 & 4.3 & 1.47 & 0.897 & & \\
\hline & PE3 & 4.03 & 1.44 & 0.848 & & \\
\hline \multirow[t]{3}{*}{ Subjective norm } & SN1 & 3.02 & 1.36 & 0.873 & 0.94 & 0.7921 \\
\hline & SN2 & 3.19 & 1.36 & 0.906 & & \\
\hline & SN3 & 2.96 & 1.32 & 0.915 & & \\
\hline \multirow[t]{3}{*}{ Perceived behavior control } & $\mathrm{PBC} 1$ & 4.94 & 1.38 & 0.917 & 0.99 & 0.8513 \\
\hline & $\mathrm{PBC} 2$ & 4.99 & 1.54 & 0.965 & & \\
\hline & $\mathrm{PBC} 3$ & 4.85 & 1.58 & 0.937 & & \\
\hline
\end{tabular}

Table 3. Correlation Matrix and Discriminant Assessment

\begin{tabular}{lcccccccc}
\hline & 1 & 2 & 3 & 4 & 5 & 6 & 7 & 8 \\
\hline 1.Continuance intention & 0.768 & & & & & & & \\
2.User satisfaction & 0.415 & 0.808 & & & & & & \\
3.Perceived usefulness & 0.387 & 0.682 & 0.795 & & & & & \\
4.Confirmation & 0.399 & 0.591 & 0.627 & 0.812 & & & & \\
5.Attitude & 0.178 & 0.199 & 0.349 & 0.405 & 0.746 & & & \\
6.Perceived ease & 0.304 & 0.54 & 0.627 & 0.566 & 0.313 & 0.789 & & \\
7.Subjective norm & 0.284 & 0.52 & 0.607 & 0.536 & 0.283 & 0.759 & 0.675 & \\
8.Perceived behavior control & 0.079 & 0.339 & 0.341 & 0.348 & 0.288 & 0.421 & 0.693 & 0.58 \\
\hline
\end{tabular}

\section{Conclusion}

We have examined the effects of satisfaction, perceived usefulness, confirmation, perceived ease of use, attitude, subjective norm, and perceived behavioral control on the older adults' adoption and continuance intention of SNSs. The results of the study show that satisfaction is the strongest predictor of older adults' continuance intention, followed by perceived usefulness, attitude, confirmation, perceived ease of use and subjective norm. Perceived behavioral control is weaker predictor. The satisfaction-intention link has previously been validated in consumer behavior research under the product and service contexts $[2,6]$. Further, satisfaction may be the key to explaining why the older adults discontinue using SNSs after their initial acceptance. Because satisfaction was the strongest predictor of continuance intention relative to the other predictors, older adults dissatisfied with SNSs may stop using it, despite having positive perceptions with regard to other elements.

In some previous TAM-based studies of IS acceptance, perceived usefulness was a stronger predictor of acceptance intention than other predictors. But, in this study, perceived usefulness was identified as a secondary determinant of continuance intention. Perceived usefulness and perceived ease of use affect older adults' preacceptance attitude. But the attitude is formed potentially through some second- 
hand information from others people, the media, advertising or other sources. These influence sources may be biased, and hence older adults' attitude potentially may be inaccurate, unrealistic, and uncertain. In contrast, post-acceptance satisfaction is grounded in users' first-hand experience, and is therefore more realistic, unbiased, and less susceptible to change. The above result implies that perceived usefulness is more closely related to acceptance intention, while satisfaction plays a more important role for continuance intention. Therefore, in order to increase the older adults' adoption and continued usage of SNSs, the practitioners should inform new (potential) users of the potential benefits of SNSs use, and educate old (continued) users on how to use SNSs effectively so as to maximize their confirmation and satisfaction.

While all the ECM variables are significant in this study, they implicitly assume that behavior is volitional. However, older adults face several new constraints and barriers different from those of young people, such as relatively low familiarity with network technology, the degradation of the physical, sensory, and cognitive capabilities (perceived behavioral control), and influence of the opinions of other important persons (subjective norms). These issues call for the inclusion of TPB in the SNSs adoption model, and both subjective norm and perceived behavioral control are verified as having a significant influence on continuance intention. This finding implies that when older adults find that people around them have adopted SNSs services, they will be more willing to use it. So, as the results of some researches [6,7], SNSs providers may use positive word-of-mouth strategy to enhance the older adults' awareness of the SNSs and promote its benefits, and they also may need to consider how to bring all those existing older adults users positive experience to remain their future acceptance, rather than rely on mass media only.

\section{Limitation}

This study attempts to develop a new theory by hybrid the ECM, TAM and TPB model, and applying them into the context of older adults' continuance intention toward SNSs. Hence, the proposed model makes an important contribution to the emerging literature on the behavior of older adults and SNSs adaption. But, this study also has several limitations. First, the work was conducted using a short-term snapshot of older adults' behavior, and additional research efforts with longitudinal studies would give a clearer picture of how the older adults and the relationships among variables change over time. Second, the use of cross-sectional survey data has limitations. Because of the time limited, the validity of the numerical example should be further researched and improved yet. Third, while this study synthesizes three theoretical perspectives to propose a new model to explain and predict older adults' continuance intention to use SNSs, other external factors may also be important in understanding older adults' continuance intention to use SNSs.

\section{Appendix A. List of Model Constructs and Items}

\section{Continuance Intention adapted from Bhattacherjee (2001)}

I will keep using the SNSs as regularly as I do now

I will frequently use the SNSs in the future

I will strongly recommend that others use it

\section{User Satisfaction adapted from Bhattacherjee (2001)}

How do you feel about your over experience with SNSs?

1. Very dissatisfied/Very satisfied.

2. Very displeased/Very Pleased.

3. Very frustrated/Very contented. 
4. Absolutely terrible/Absolutely delighted.

I am pleased with the experience of using SNSs

My decision to use SNSs was a wise one

Perceived Usefulness adapted from Davis (1992).

I find SNSs can help me contact with other people effectively

I find SNSs can help me give more useful information quickly

I find SNSs can help me perform many things more conveniently

Perceived Ease of use adapted from Davis (1992)

Learning to operate the SNSs is easy for me

It is easy for me to become skillful at using the SNSs

Overall, the SNSs is easy to use

Attitude adapted from Davis (1992)

Using SNSs is a good idea

I like using SNSs

It is desirable to use SNSs

Confirmation adapted from Bhattacherjee (2001).

My experience with using the SNSs was better than I expected

The service level provided by the SNSs was better than I expected

Overall, most of my expectations from using SNSs were confirmed

Perceived Behavioral Control adapted from Taylor and Todd (1995)

I would be able to use SNSs.

Using SNSs is entirely within my control.

I have the resources and the knowledge and ability to make to use of SNSs.

Subjective norm adapted from Taylor and Todd (1995)

People important to me support my use of SNSs

People who influence me think that I should use SNSs

People whose opinions I value prefer that I should use SNSs

\section{Acknowledgements}

This research was supported by the National Social Science Foundation of China under Grant 13BTQ059 and Technology Foundation of Jiangxi Provincial Department of Education under Grant GJJ12730 and GJJ12267.

\section{References}

[1] G. J. Yong, "Older Adults in an Aging Society and Social Computing: A Research Agenda", INTL, Journal of human-computer interaction, vol. 26, no. 11-12, (2010), pp. 1122-1146.

[2] M. T. Braun, "Obstacles to social networking website use among older adults", Computers in Human Behavior, vol. 29, (2013), pp. 673-680.

[3] A. Lenhart, "Adults and social network websites", Pew Internet and American Life Project, (2009).

[4] M. Madden, "Social networking use among those ages 50 and older nearly doubled over the past year", Pew Internet and American Life Project, (2010).

[5] N. Wagner, K. Hassanein and M. Head, "Computer use by older adults: A multi-disciplinary review", Computers in Human Behavior, , vol. 26, (2010), pp. 870-882.

[6] A. Bhattacherjee, "Understanding information systems continuance", An expectation-confirmation model, MIS Quarterly, vol. 25, no. 3, (2001), pp. 351-370.

[7] B. S. Kim, "An empirical investigation of mobile data service continuance: Incorporating the theory of planned behavior into the expectation-confirmation model", Expert Systems with Applications, vol. 37, (2010), pp.7033-7039.

[8] I. Ajzen, "The theory of planned behavior", Organizational Behavior and Human Decision Processes, vol. 50, no. 2, (1991), pp. 179-211.

[9] F. D. Davis, R. P. Bagozzi and P. R. Warshaw, "Extrinsic and intrinsic motivation to user computers in the workplace", Journal of Applied Social Psychology, vol. 22, no. 14, (1992), pp. 1111-1132.

[10] S. Taylor and P. A. Todd, “Assessing IT usage: The role of prior experience”, MIS Quarterly, vol. 19, no. 4, (1995), pp. 561-570.

[11] T. J. Larsen, "The role of task-technology fit as users'motivation to continue information system use", 
Computers in Human Behavior, vol. 25, (2009), pp. 778-784.

[12] M. C. Lee, "Explaining and predicting users'continuance intention toward e-learning: An extension of the expectation-confirmation model”, Computers \& Education, vol. 54, (2010), pp. 506-516.

\section{Author}

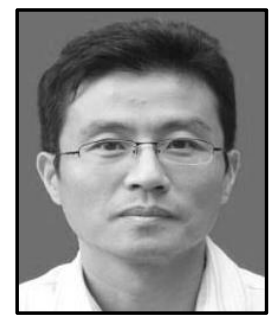

Wei Liu was born in ShangHai china, in May 1971. He received his $\mathrm{PhD}$ in management information systems from School of Economics \& Management, BeiHang University, China, in 2007. His current research interests include social network services, mobile commerce and adoption behavior.

$\mathrm{He}$ is an IS associate professor and Institute of Information Resources Management Faculty Fellow at School of Information Technology, Jiangxi University of Finance \& Economics, China. 